\title{
Mitochondrial alterations during oxidative stress in chronic obstructive pulmonary disease
}

\author{
This article was published in the following Dove Press journal: \\ International Journal of COPD \\ 13 April 2017 \\ Number of times this article has been viewed
}

\section{Ying Jiang \\ Xiaoqin Wang \\ Daode $\mathrm{Hu}$}

Department of Clinical Pharmacology, Shanghai General Hospital, Shanghai Jiao Tong University School of Medicine, Shanghai, People's Republic of China
Correspondence: Daode Hu Department of Clinical Pharmacology, Shanghai General Hospital, 100 Haining Road, Hongkou, Shanghai 200080,

People's Republic of China

Tel +8602I 63240090

Email shanghaiyao@sina.com

\begin{abstract}
The high incidence of chronic obstructive pulmonary disease (COPD), one of the most prevalent diseases worldwide, has attracted growing attention. Cigarette smoking is considered a major contributory factor in the pathogenesis and progression of COPD due to the tremendous oxidative burden that it causes, which induces an oxidant/antioxidant imbalance. Excessive oxidation induced by the excessive generation of mitochondrial reactive oxygen species disturbs the antioxidant systems and plays an important role in triggering and promoting chronic inflammation of airways. Given that mitochondria is one of the main sites of reactive oxygen species production by the oxidative phosphorylation process, oxidative stress may affect mitochondrial function by changing its structure and morphology and by affecting a series of mitochondrial proteins. In particular, PTEN-induced putative kinase 1/Parkin and p62 play critical roles in mitophagy. During the process, the Akt ubiquitin E3 ligase is an important mediator associated with cigarette smoke exposure-induced pulmonary endothelial cell death and dysfunction. Thus, understanding the underlying mechanisms of the signaling pathway may provide important information regarding the therapeutic treatment of COPD by application of alternative PTEN-induced putative kinase 1 targets or ubiquitin E3 ligase.
\end{abstract}

Keywords: chronic obstructive pulmonary disease, reactive oxidative stress, reactive nitrogen stress, mitochondrial fission/fusion, mitophagy

\section{Introduction}

Chronic obstructive pulmonary disease (COPD), an inflammatory disease with rising incidence worldwide, is characterized by sustained airflow limitation and is always associated with pulmonary arterial hypertension, hypoxemia, and hypercapnia, which significantly increases the likelihood of cardiopulmonary complications and aggravates the patients' conditions. A survey showed that about 65 million people have COPD globally, and almost 3 million people, majorly from low- and middle-income countries, died of complications associated with COPD in $2005 .^{1}$

With regard to the causes of the development of COPD, various studies have proven that oxidative stress induced by reactive oxygen species (ROS) plays a major role in triggering and promoting chronic inflammation of airways and imbalances in protease/antiprotease activity. ${ }^{2}$ Cigarette smoking is one of the most important risk factors that contain a large amount of ROS that induce an oxidant burden in smokers, ${ }^{3-7}$ affecting the oxidant-antioxidant mechanisms in the development of COPD. ${ }^{8}$ Exposure to cigarette smoke induces the production of endogenous ROS as a byproduct of functional electron transfer chain in the mitochondria. ${ }^{9}$ Mitochondria is a crucial semiautonomous cellular organelle housing the metabolic processes of oxidative phosphorylation and fatty acid metabolism, and is also a tool bridging signal transduction between the nucleus and extracellular threats. Despite the fact that 
mitochondria is one of the main sources of endogenous ROS, mitochondrial dysfunction has been found in oxidant-induced lung damage due to the susceptibility of mitochondrial DNA to oxidative damage, which consists of a reduction in mitochondrial biogenesis. ${ }^{10,11}$ Peroxisome proliferator-activated receptor $\gamma$ coactivator $1 \alpha(\mathrm{PGC}-1 \alpha)$, a central integrator of multiple signaling pathways, has been demonstrated to play a key role in the regulation of mitochondrial biogenesis and oxidative stress by a mitochondrial transcription factor $\mathrm{A}$ (TFAM). ${ }^{12}$ These signaling factors regulating mitochondrial biogenesis are increased in COPD muscle, leading to a low muscle oxidative capacity in COPD. ${ }^{10,13}$

Oxidative stress affects not only mitochondrial biogenesis, but also the mitochondrial quality control in the pathogenesis of lung diseases. ${ }^{14}$ A previous study indicated that ROS induced by cigarette smoke were involved in the alterations in mitochondrial network morphology. ${ }^{15}$ Additionally, in the airway smooth muscle (ASM) from patients with an airway disease, mitochondria exhibited substantial morphologic defects and the balance between mitochondrial fission and fusion was disturbed. Similar alterations have also been observed in the mitochondria in airway epithelial cells of COPD patients exposed to chronic cigarette smoke. ${ }^{16}$ Overall, mitochondria seem to play an important role in different aspects of cellular structure and function, as well as in ROS induced by alterations of mitochondrial morphology. However, the molecular mechanisms between the mitochondria-induced ROS and the pathologic process of COPD have not been fully understood. Thus, this review aims to discuss how oxidative stress affects the progression and pathogenesis of COPD, and the corresponding alterations in mitochondria-mediated structures in response to oxidative stress have also been reviewed.

\section{Source of ROS}

Oxidation is a chemical process in which electrons are removed from molecules; during the process, the leaked electrons contribute to generation of some highly reactive free radicals. Generally speaking, the accumulated ROS are derived in two ways: exogenous and endogenous ROS (Figure 1). Exogenous ROS primarily originate from inhaled toxic gases or environmental pollutants such as car exhaust fumes, occupational exposure to dusts and cigarette smoking.

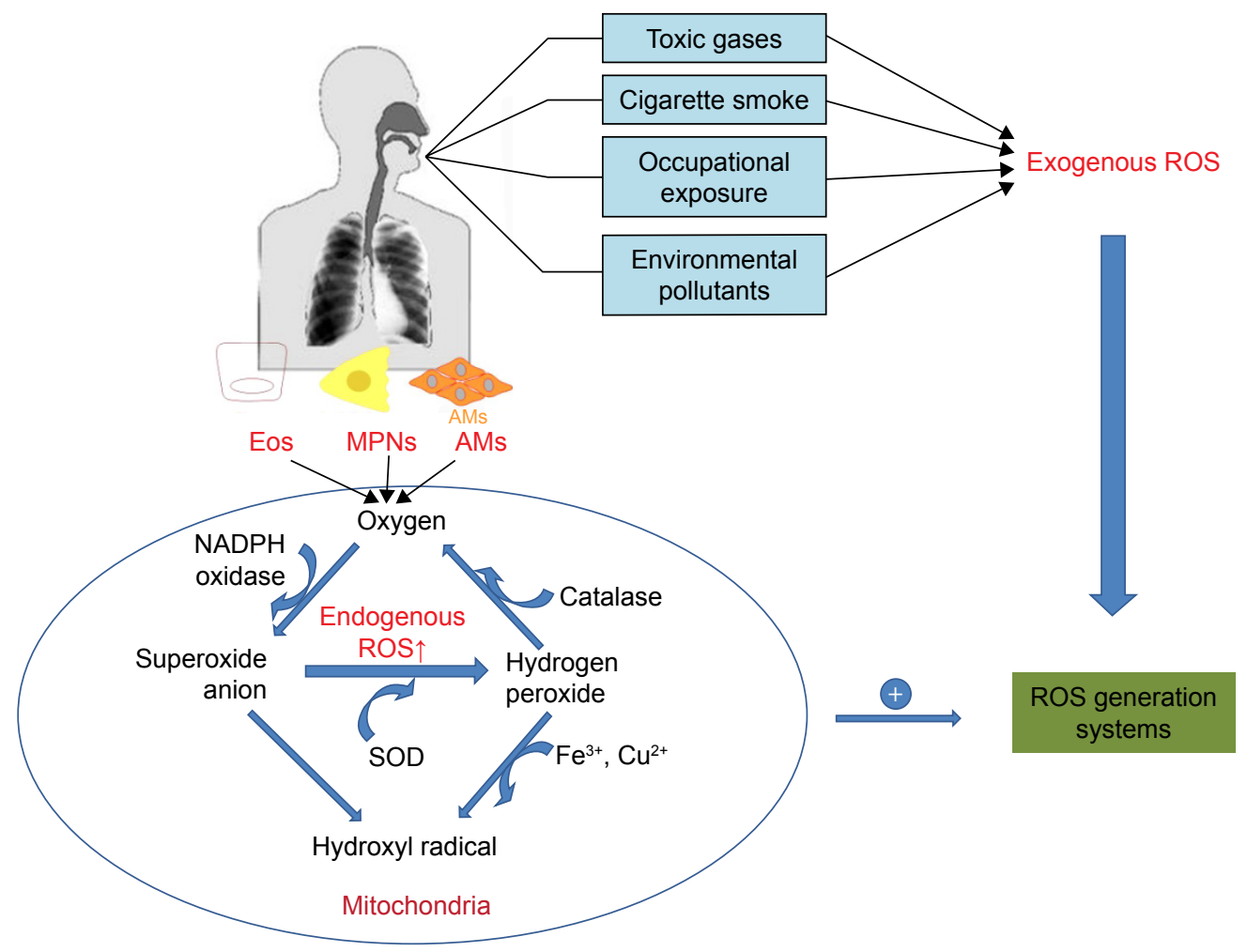

Figure I Sources of ROS in vivo.

Notes: Toxic gas, cigarette smoke, occupational exposure and environmental pollutants are the major sources of exogenous ROS that result in lung injuries in COPD. Alveolar macrophages, eosinophils and epithelial cells are damaged by interacting with inhaled oxidants, activating various relevant enzymes such as SOD and catalase to convert the oxygen inhaled to another forms of ROS, which contributes to the endogenous ROS in vivo. Initiating by endogenous and exogenous stimulus, the ROS-generating systems can be activated via the redox-sensitive pathway.

Abbreviations: COPD, chronic obstructive pulmonary disease; ROS, reactive oxidative specis; SOD, superoxide dismutase; Eos, eosnophils; MPNs, polymorphonuclear cells; AMs, alveolar macrophages. 
The sources of endogenous ROS include mitochondrial respiration, peroxisomes, the NADPH oxidase (NOX) system and inflammatory cells, ${ }^{17}$ while the oxidative phosphorylation process in the mitochondria is one of the major sources of endogenous ROS. ${ }^{9,18}$

Peroxisome is a source of endogenous ROS, especially hydrogen peroxide $\left(\mathrm{H}_{2} \mathrm{O}_{2}\right)$ and superoxide anions $\left(\mathrm{O}_{2}^{-}\right)$. For example, a heme-containing peroxidase in neutrophils and monocytes, myeloperoxidase, correlates with an increased level of ROS and chronic inflammation in COPD patients. ${ }^{19,20}$ Inflammatory cells also contribute to the formation of endogenous ROS under the catalysis of intracellular enzymes such as NOXs, ubiquinone reductase and cytochrome oxidase, while NOXs have long been recognized to be one of the primary generators of ROS within the lung and play pivotal roles in the pathogenesis of a number of diverse chronic lung disorders. ${ }^{21,22} \mathrm{NOX}$ enzymes catalyze the conversion of $\mathrm{O}_{2}$ into $\mathrm{O}_{2}^{-}$, which is a reactive radical characterized by its speedy interaction with intracellular macromolecules (lipids, proteins and DNA). There are seven members of NOXs: NOX1-5, dual oxidase DUOX1 and DUOX2. The expression levels of DUOX1 and DUOX2 that are primary expressed in the barrier epithelia were found to be low in the airway epithelium of former smokers with mild/moderate COPD. ${ }^{23,24}$ NOX1 mRNA expression was increased in patients with COPD, which contributes to rhinovirus infection-related exacerbations. ${ }^{25}$ Pulmonary vascular endothelial cells have been shown to produce ROS in the presence of NOX $2 .{ }^{26}$ NOX3 in the lung was found to be associated with lung destruction and emphysema in aged mice with TLR4 deletion, but these effects were reversed on treatment with NOX inhibitors. ${ }^{27}$ The ability of NOX4 to generate ROS has also been verified. An increasing number of researches have revealed that an increased expression of NOX4 was observed in pulmonary diseases. ${ }^{28-31}$ Recently, Hollins et al found that the expression of NOX4 was increased in ASM in COPD both in vivo and in primary cells in vitro. ${ }^{32}$ Liu et al also showed NOX4 protein was increased in the ASM, and its expression correlated positively with disease severity and inversely correlated with the pulmonary functions in COPD patients. ${ }^{33}$ Inhibition of NOX4 expression increased the mitochondrial TFAM level, leading to stimulation of mitochondrial biogenesis. ${ }^{12}$ Oxidative stress induced by abundance of ROS can affect the organelles and disrupt cellular homeostasis.

Mitochondria are one of the main sources of production of ROS in eukaryotic cells. The production of ROS is a consequence of electron leakage occurring in the mitochondrial electron transport chain. During aerobic respiration, highenergy electrons pass through electron carriers in the respiratory chain complexes and react with molecular oxygen to form $\mathrm{O}_{2}^{-}$, primarily at complexes I and III. This process is responsible for the generation of adenosine triphosphate (ATP), which promotes pumping of protons from the mitochondrial matrix into the intermembrane space, creating a measurable electrochemical proton gradient. However, protons are pumped back into the matrix in the presence of ATP synthase and react with the $\mathrm{O}_{2}^{-}$in a reaction catalyzed by superoxide dismutase, contributing to the formation of hydrogen peroxide. ${ }^{34}$ Superoxide at a high concentration causes oxidative damage. The formed $\mathrm{H}_{2} \mathrm{O}_{2}$ may give rise to hydroxyl radicals or is decomposed into water and oxygen by catalase. Therefore, the presence of $\mathrm{O}_{2}^{-}$plays an important role in the formation of other forms of ROS. Excessive levels of mitochondrial ROS degrade the mitochondrial function and lead to the collapse of electrochemical gradient, which leaves the cell more susceptible to ROS-mediated injury. ${ }^{35}$ Given that oxidative stress in COPD patients is a prevailing phenomenon, we next reviewed how oxidative stress induced by excessive ROS plays a role in the pathogenesis of inflammation in COPD.

\section{The role of oxidative stress in COPD pathogenesis}

Minutoli et $\mathrm{al}^{36}$ and Park et $\mathrm{al}^{37}$ suggested that accumulation of mitochondrial ROS activates inflammatory pathways in various tissues by interfering with mitochondrial quality control. Evidence shows that excessive mitochondrial ROS causes oxidative damage, which was reflected by the increased levels of damaged proteins, DNA and lipids in the mitochondria, as well as inflammatory markers within the cells in COPD patients. ${ }^{38}$ Actually, ROS exhibit their toxic properties under specific conditions when the antioxidant system fails to eliminate excessive free radicals. In this case, gaseous-phase ROS in inhaled irritants cause direct damages to airway epithelial cells and their plasma cell membranes when its level exceeds the scavenge ability of the cellular anti-oxidative system. ${ }^{39}$ Thus, antioxidant defense systems, including the nuclear factor-erythroid2-related factor 2 (Nrf2)-mediated antioxidant system (Figure 2) and the phase II detoxifying enzymes, are attenuated by excessive accumulation of ROS. Denuded epithelia are always observed in COPD, increasing the possibility that the ROS can reach the ASM and promote the proliferation of ASM (Figure 3), leading to thickening of the airway walls and an accelerated decline in lung function. ${ }^{40}$ 


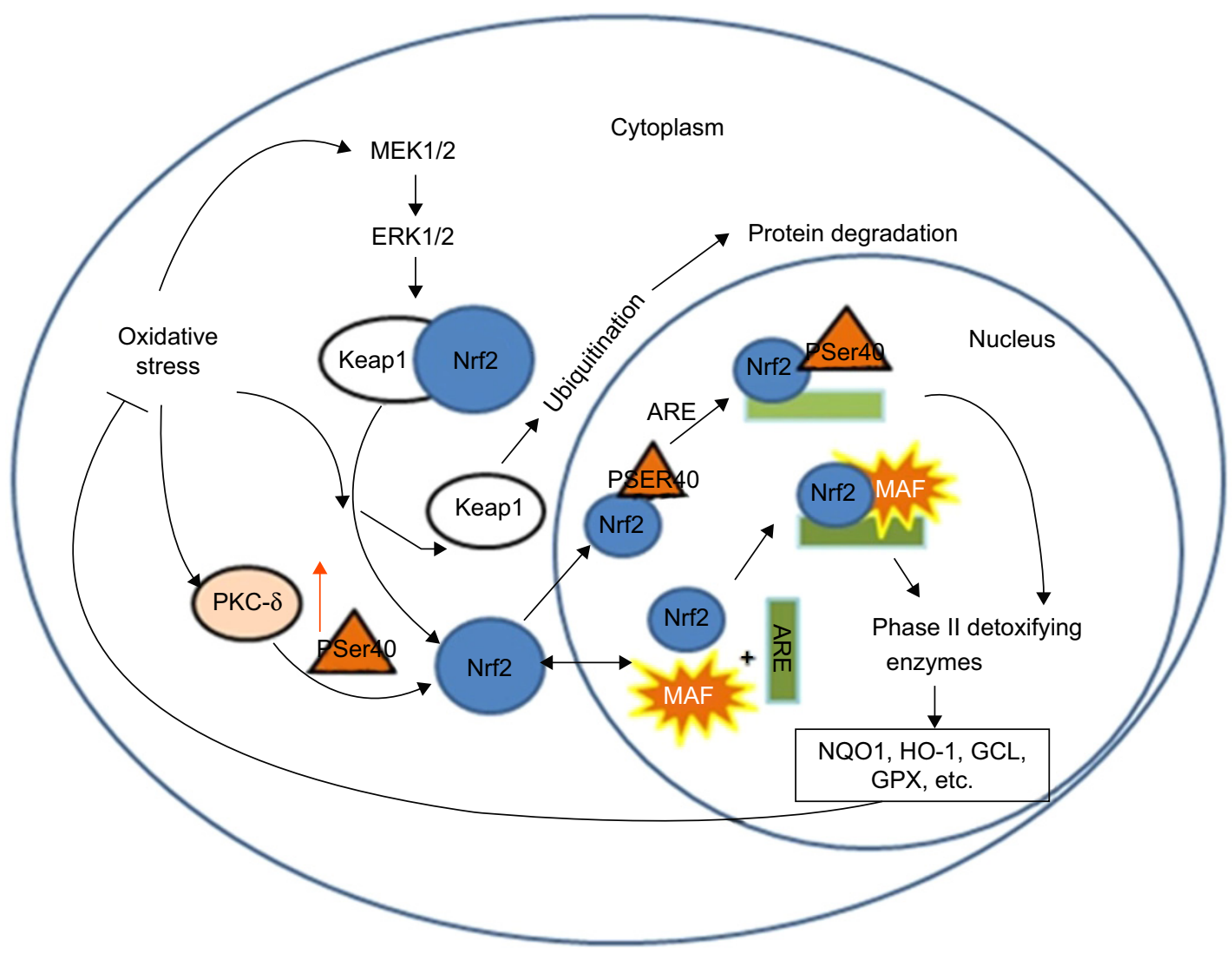

Figure 2 Antioxidant mechanisms of stress sensing in Nrf2-mediated system. Abbreviation: Nrf2, nuclear factor-erythroid-2-related factor 2.

Inflammatory cells such as $\mathrm{CD}^{+} \mathrm{T}$ lymphocytesneutrophils and alveolar macrophages are stimulated on exposure to oxidative stress, which affects the pulmonary epithelial cells. ${ }^{41}$ One of the most obvious alterations in the inflammatory process is the imbalance of the protease/ antiprotease system within the respiratory system, which promotes the release of the proteolytic enzymes of neutrophils and macrophages (matrix metalloproteinase-12), thus contributing to the degradation of their natural inhibitors. Consequently, the pulmonary parenchyma is destroyed, which accounts for the emphysema. During this process, accumulation of neutrophil elastase, a potent serine proteinase and a key effector that is most likely responsible for the emphysema, has been found in COPD patients. ${ }^{42}$ Moreover, neutrophil elastase upregulates the levels of interleukin (IL)-8 and mucin $5 \mathrm{AC}$ that is a glycoprotein linked to mucus hypersecretion in the pulmonary tract. In bronchial epithelial and alveolar cells, ROS also induce the gene expression of inflammatory mediators such as tumor necrosis factor (TNF)- $\alpha$, which in turn activates the transcription of IL-8, matrix metalloproteinase-9, mucin $5 \mathrm{AC}$ as well as interferon- $\gamma^{4}{ }^{43-46}$ Several mechanisms such as TNFRs, NLRP3 inflammasome signals and MAPK are responsible for the mitochondrial ROS-mediated inflammatory responses. Bulua et al stated that mitochondrial ROS promote the production of proinflammatory cytokines including IL-6 and TNF in the cells from patients with TNFR1-associated periodic syndrome. ${ }^{47}$ Levels of IL-1 $\beta$ and IL-18 are increased by the NLRP3 pathway. However, redox-sensitive transcription factors including NF- $\mathrm{KB}$ and AP-1 regulate these inflammatory genes' expression.

\section{Alterations of mitochondrial morphology in COPD}

Recently, alterations in mitochondrial morphology are thought to be involved in oxidative stress in COPD pathology. The structural changes of mitochondria are closely linked to oxidative stress-mediated signaling. Cigarette smoke induced mitochondrial fragmentation and damaged their networked morphology in nonasthmatic human ASM cells, which depended on the level of oxidative stress. ${ }^{16}$ However, cigarette smoke-triggered oxidative stress at a mild level induced mitochondrial hyperfusion in alveolar epithelial cells, which renders the cell more vulnerable to additional stress. ${ }^{48}$ Furthermore, cigarette smoke-induced mitochondrial hyperfusion diminished mitochondrial quality control and impaired 


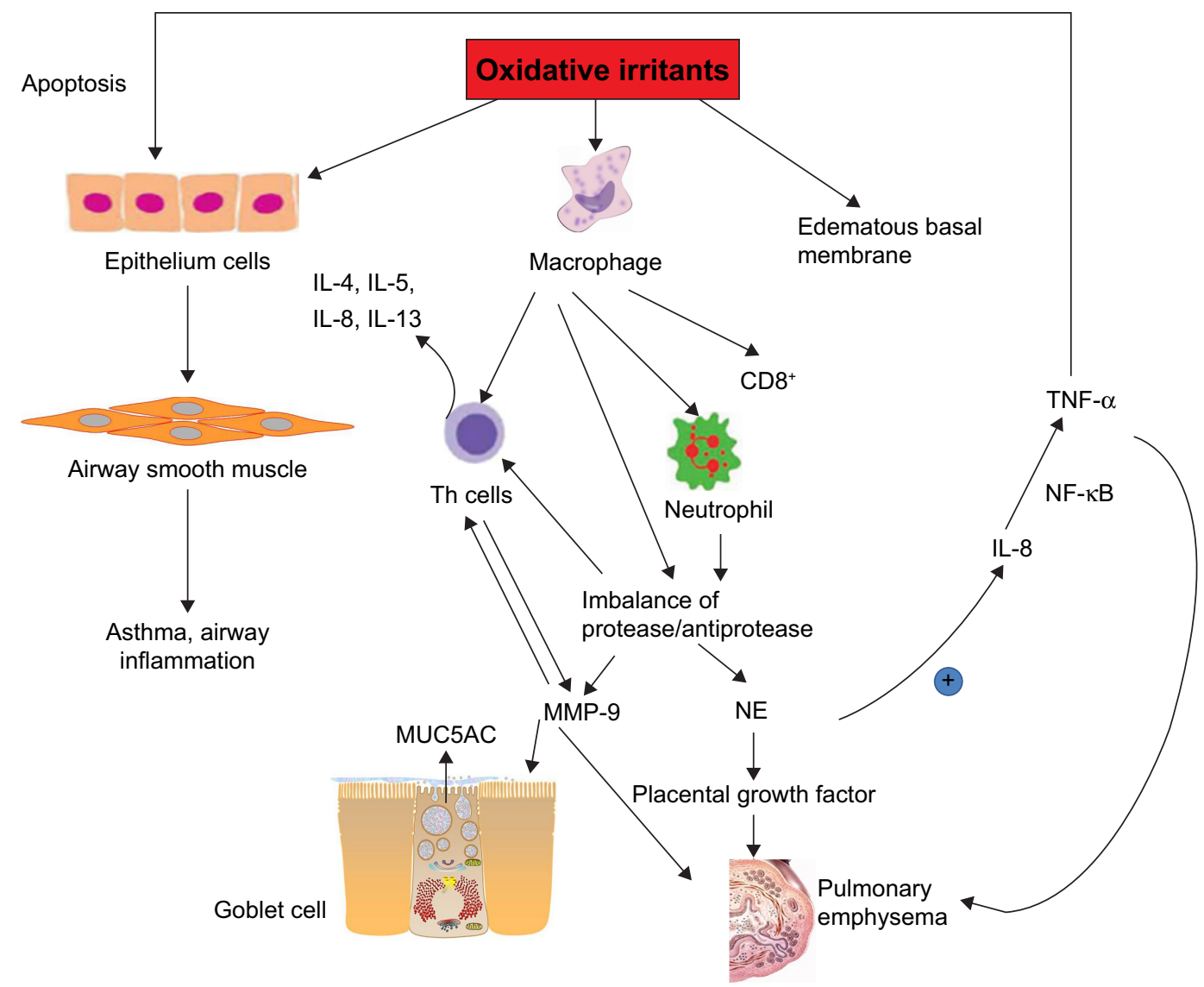

Figure 3 Mechanisms of oxidative stress injuries in COPD.

Notes: Oxidative irritants including tobacco smoke directly affect the epithelial cells, macrophages, and edematous basal membrane. The ASM markedly increases under excess exposure of ROS. The activated macrophage passes oxidative signaling to neutrophils and Th cells, which causes imbalance in protease/antiprotease system and overexpressions of MMP- 9 as well as NE. The increased expression of NE induces the TNF- $\alpha$ by the NF- $\kappa B$ signaling pathway, which accelerates apoptosis of epithelial cells. Abbreviations: ASM, airway smooth muscle; COPD, chronic obstructive pulmonary disease; IL, interleukin; MMP, matrix metalloproteinase; MUC5AC, mucin 5AC; $\mathrm{NE}$, neutrophil elastase; ROS, reactive oxygen species; TNF, tumor necrosis factor.

cellular stress resistance and cellular senescence, contributing to age-related COPD pathogenesis.

Thus, it is necessary to understand how the mitochondrial morphology is altered in response to the cellular stress induced by ROS in the progression of COPD.

To provide protection against severe cellular stress, mammalian mitochondria are not static structures, but undergo frequent shape changes to form tubular, reticular or networked morphology by changing mitochondrial fission/ fusion. Both mitochondrial hyperfusion and mitochondrial fragmentation are a part of the mitochondrial quality control to maintain robust mitochondrial function for impaired mitochondria, while mitochondrial fusion is an adaptive stress-resolving mechanism that involves exchanging the damaged mitochondrial DNA, lipids or proteins with those of healthy mitochondria. ${ }^{49}$

Mitochondrial fusion leads to elongated mitochondria in order to eliminate defective mitochondrial components. ${ }^{50}$ During this process (Figure 4), three large guanosine triphosphatases (GTPases), including mitofusion (Mfn) 1 and 2 embedded in the outer membrane of mitochondria and fusion protein (optic atrophy protein 1 [Opa1]) on the inner membrane of mitochondria, are of particular interest in morphologic maintenance. In fact, Opa1 and Mnfs share a reciprocal relationship. The evolutionarily conserved GTPases, Mfn1 and Mfn2, are responsible for outer mitochondrial membrane fusion, ${ }^{51}$ while the pro-fusion effect of Mfn1 is promoted by the binding to guanine nucleotides to protein- $\beta$ subunit 2 (Gß2), thus facilitating and stabilizing clustering of Mfn1 on the outer mitochondrial membrane. ${ }^{52}$ Furthermore, the effects of Mfn2 are attributed to the expression of PGC1- $\alpha$ and PGC1- $\beta .^{53}$ The dynamin-related GTPase, Opa1, mediates inner membrane fusion, and depletion of this protein results in severely reduced respiratory efficiency. ${ }^{54}$ The effects of these three proteins have been proven by mouse knockout studies, which provided clear evidence of reduced mitochondrial fusion as an adaptive mechanism of resolving cellular stress in the cells. ${ }^{55-57}$ Hoffmann et al ${ }^{16}$ suggested that cigarette smoke induced morphologic changes in the COPD epithelium by significantly increasing the expression 


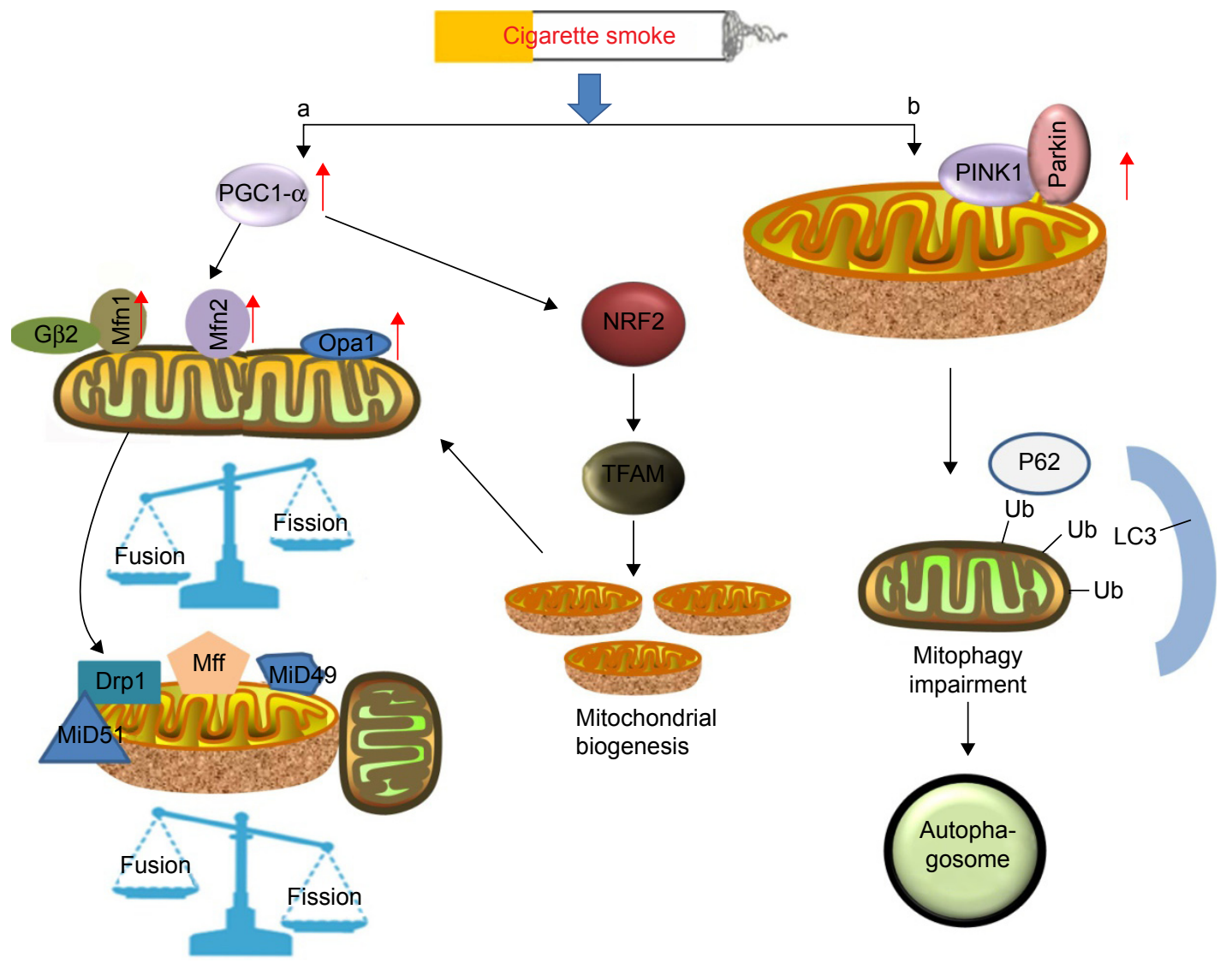

Figure 4 Schematic diagram shows that cigarette smoke induces mitochondrial fusion/fission and mitophagy.

Notes: (a) Cigarette smoke causes mitochondrial fusion in response to mild stress by increasing the accumulation of Mfnl/2 and Opal. Mitochondrial fission occurs in response to increased ROS. (b) When the depolarized or fragmented mitochondria are unable to fuse back into the functional pool of mitochondria, PINKI accumulates on the mitochondrial membrane and recruits the E3 ligase Parkin. Parkin conjugating with Ub mediates the degradation of mitofusins I and 2 . P62 binds to the outer membrane Ub to facilitate mitochondria targeting to LC3.

Abbreviations: Mfn, mitofusion; Opal, optic atrophy protein I; PINKI, PTEN-induced putative kinase I; ROS, reactive oxygen species; Ub, ubiquitin

of Mfn1/2 and Opa1, which were accompanied by increased levels of the proinflammatory mediators such as IL-8, IL-6 and IL-1 $\beta$. Such a phenomenon of mitochondrial hyperfusion was also observed in the alveolar epithelial cells on exposure of cigarette smoke, which renders the cells more vulnerable to additional stress. ${ }^{48}$ Furthermore, cigarette smoke-induced mitochondrial hyperfusion diminished mitochondrial quality control and impaired cellular stress resistance and cellular senescence, contributing to age-related COPD pathogenesis. Ahmad et al have shown that cigarette smoke extract induced elongated mitochondria and reduced ATP levels in lung epithelial cells and fibroblasts. ${ }^{58}$ Mitochondrial fusion is a common phenomenon in mitochondria exposed to mild oxidative stress, whereas mitochondrial fission accelerates cellular necroptosis. ${ }^{15}$

Accordingly, mitochondrial fission is highly correlated with cell apoptosis and mitophagy. Mitochondrial fission is achieved by phosphorylation of Drp1 at Ser616, which promotes the recruitment of Drp1 from the cytosol to the mitochondrial surface, by human fission protein-1, mitochondrial fission factor and mitochondrial dynamics proteins of 49 and 51..$^{14,54}$ The possible mechanism indicates that oxidative stress increases the expression of the TFAM protein, thus triggering mitochondrial fission and loss by enhancing Drp1 translocation from the cytosol. Cigarette smoking-induced mitochondrial ROS accelerates the course of phosphorylation of Drp1. ${ }^{15}$ However, inhibition of Drp1 significantly increases Bad S112 phosphorylation, which acts against fission-mediated apoptotic cell death. ${ }^{59}$ Additionally, a prolonged exposure of cigarette smoke in COPD patients also significantly induces the expression of Fis 1 and decreases the levels of Mfn1/2 and Opa1 on the airway epithelial mitochondria, ${ }^{59}$ indicating that ROS contribute to mitochondrial fission. Mitochondrial fragmentation has further been described in the ASM cells of patients with COPD. ${ }^{60}$ ASM cells cultured from a restricted group of COPD patients showed a collapsed mitochondrial membrane potential, an increased expression of mitochondrial fission protein and 
reduced respiratory complex protein, and accumulation of mitochondrial ROS, which contribute to the development of airway hyperresponsiveness and lung inflammation. ${ }^{60}$ Moreover, Aravamudan et al also demonstrated that cigarette smoke induced mitochondrial fragmentation and damaged their networked morphology by increased expression of Drp1 and decreased Mfn2 in nonasthmatic human ASM cells, and this process involved ROS. ${ }^{15}$ Recently, Wang et $\mathrm{al}^{61}$ demonstrated that stress-induced mitochondrial fission and apoptosis were mediated by cyclin C-Cdk8 by enhancing the association between Drp1 and mitochondrial fission factor, which stabilizes their retention on the mitochondria in mouse embryonic fibroblasts. However, whether the same effects of $\mathrm{C}-\mathrm{Cdk} 8$ are linked to lung disease remains unknown and deserves further investigation.

\section{Mitophagy in COPD}

Oxidative stress impairs mitochondrial function. When the depolarized or fragmented mitochondria are unable to fuse back into the functional pool of mitochondria, mitophagy will take place as a protective mechanism by degrading the cellular components to prevent spreading of damage in the mitochondrial network. ${ }^{62}$ The formation of autophagosomes is a consequence of this process. ${ }^{18}$ Mitophagy plays a potentially protective role in removal of mitochondria damaged by cigarette smoke. ${ }^{63}$ A cytosolic E3 ubiquitin ligase Parkin and the PTEN-induced putative kinase 1 (PINK1) that is an upstream regulator of Parkin are stimulated in this process. ${ }^{62,64}$ When the mitochondria are depolarized and fail to regain their membrane potential to protect themselves from detrimental effects, the process of mitophagy begins. ${ }^{62}$ Recent observations have shown that cigarette smoke exposure induces mitophagy, which contributes to overall mitochondrial quality in the epithelial cells and is likely initiated by PINK1 stabilization. The PINK1-PARK2-mediated pathway may account for mitophagy. ${ }^{65}$ Knockdown of PINK1 and PARK2 results in enhanced mitochondrial ROS production and cellular senescence in human bronchial epithelial cells, with insufficient mitophagy of the cells. These findings suggest that defective mitochondria in a cell undergo mitophagy in a PINK1-dependent manner and that PINK is the initiator of this process. ${ }^{66}$

In terms of mechanism (Figure 4), PINK1/Parkin-mediated mitophagy is a complex process involving ubiquitination and autophagy. ${ }^{67}$ Upon dissipation of the mitochondrial membrane potential, Parkin translocates to depolarized mitochondria through phosphorylation at Ser65 in the ubiquitin-like domain (Ubl) by PINK1. ${ }^{68}$ Subsequently, numerous outer mitochondrial membrane proteins are ubiquitinated by Parkin, which in turn triggers accumulation of other proteins on the mitochondrial surface. ${ }^{69,70}$ In a mechanistic study of the damaged mitochondria, two distinct polyubiquitin chains, linked through K27 and K63 of ubiquitin, were reported to be formed at clustered mitochondria in the presence of Parkin. ${ }^{69}$ Accompanied with the formation of the specific polyubiquitin chains, a molecular weight shift of voltage-dependent anion channel 1 (VDAC1) seemed to be correlated with an increased level of PINK1, indicating that VDAC1 probably participated in PINK1/Parkin-dependent mitophagy. Indeed, it was identified that phosphorylation of VDAC1 is necessary in Parkin-mediated mitophagy, during which the adapter protein p62 exerts a pivotal role in the clearance of damaged mitochondria since Parkin-dependent mitophagy requires p62/SQSTM1/sequestosome-1 recruitment to the clustered mitochondria for final clearance. ${ }^{70}$ However, the underlying mechanisms of p62-mediated clearance in this pathway have not been fully understood. Previous studies have indicated that the autophagic clearance was regulated by Ser403 phosphorylation of p62, a step promoted by casein kinase 2 or TANK-binding kinase $1 .^{71,72}$ Ro et al identified Unc-51-like protein kinase 1 as a novel p62 ser403 kinase, while Sertrin2 is a promoter of Ser403 phosphorylation. ${ }^{73}$ Recently, multiple phosphorylation sites of $\mathrm{p} 62$ in ubiquitin association domains have been recognized, ${ }^{74}$ of which phosphorylation of Ser409 by Unc-51-like protein kinase 1 significantly increases the binding affinity of p62 to ubiquitin under proteotoxic conditions. Thus, the phosphorylated p62 linked with the damaged mitochondria by ubiquitin is recognized by the receptors on an autophagosome.

P62 also has an impact on the elimination of toxic proteins. Cigarette smoking-related COPD is known to induce an imbalance in protein homeostasis in the mitochondria, which initiates aggregation of ubiquitinated proteins, ${ }^{75}$ which in turn promotes the accumulation of PINK1 and leads to mitophagy. Thus, autophagy is responsible for the elimination of these aggregative proteins. The targeting cargo of mitophagy to autophagosomes is recognized by a body of ubiquitin-binding autophagic adaptors (eg, p62/SQSTM1/sequestosome-1), which connect the ubiquitin system and the autophagic mechanism, promoting the binding of autophagic substrates and the small ubiquitin-like modifiers that belong to the autophagy-related 8/LC3 family located on the membrane of autophagosomes. ${ }^{76}$ Furthermore, the cargo receptor for autophagic degradation, p62, has also been identified as a target gene for the transcription factor $\mathrm{Nrf} 2$ and creates a positive feedback loop for oxidative stress by activation of 
antioxidant response. ${ }^{77}$ Another study indicated that Nrf2 knockout in the Staphylococcus aureus sepsis model induced dysregulated autophagy of the alveolar cells, which led to accumulation of p62 and increased colocalization of LC3 to mitochondria. ${ }^{78}$ Thus, Nrf2-dependent mitophagy or p62targeted phosphorylation may provide a potential novel drug target for oxidative stress-related lung disease. Additionally, recent studies have indicated that mitophagic clearance also aggravates the pathogenesis of COPD. Significant accumulation of polyubiquitinated proteins and p62 was detected in the lungs when exposed to cigarette smoke extract, which accelerated lung aging and COPD-emphysema exacerbations. ${ }^{79}$ P62 as an impaired autophagy marker showed an increased level in aggresome bodies in the cells exposed to cigarette smoke extract. Recently, PINK-induced mitochondrial instability was found to be correlated with Fbxo15, an ubiquitin E3 ligase component. ${ }^{80}$ The interaction between PINK1 and Fbxo15 triggers polyubiquitination and proteasomal elimination of cardiolipin synthase, thus impairing the mitochondrial membrane. ${ }^{80}$ Mitophagy also occurred in emphysema and airway disruption, during which process the Akt ubiquitin E3 ligase mitochondrial E3 ubiquitin protein ligase 1 was identified as a novel mediator associated with cigarette smoking-induced pulmonary endothelial cell death and mitochondrial dysfunction. ${ }^{81}$ The level of mitochondrial E3 ubiquitin protein ligase 1 was noticeably elevated after exposure to cigarette smoke, potentially contributing to endothelial cell death and dysfunction in the lungs of smokers. Thus, we can presume that the alternative PINK targets or ubiquitin E3 ligase may have a potential application in the therapeutic treatment in COPD, and further investigations are necessary to explore the effects of mitophagy in the pathogenesis of COPD.

\section{Conclusion}

Oxidative stress is involved in the pathogenesis and progression of COPD and is also of great importance in the initiation of inflammation. It contributes the most to COPD by disturbing the antioxidant system and the balance of oxidant/ antioxidant within the cells, accompanied by an increased risk of comorbid diseases or inflammation. As one of the main producers of ROS, mitochondria undergo a series of changes in morphology. These alterations, such as mitochondrial fission/fusion or mitophagy, are stress reactions in response to excessive oxidation, which provides a train of thought of alleviating partial injuries of cells by targeting certain molecules. Therapies targeting enhancement of antioxidants' ability seem to be able to reduce COPD exacerbations by modulating the oxidative stress. However, untargeted antioxidants such as vitamin E and NAC lack beneficial effects sometimes or may even worsen the disease. Mitochondria-targeted antioxidants such as Mito-TEMPO have been validated for their effective role in restoring impaired mitophagy and in delaying cellular senescence in Parkin-overexpressing cells. Thus, further research that focuses on mitochondria should be carried out because it may provide important information on the potential therapeutic strategies for the treatment of COPD patients.

\section{Acknowledgments}

The authors gratefully acknowledge the financial support for this study provided by the Shanghai Committee of Science and Technology (16401901400), the Health Bureau of Shanghai, China (2011ZJ021) and the State Key Laboratory of Clinical Pharmacology Department of Shanghai General Hospital.

\section{Disclosure}

The authors report no conflicts of interest in this work.

\section{References}

1. Koul PA. Chronic obstructive pulmonary disease: Indian guidelines and the road ahead. Lung India. 2013;30(3):175-177.

2. Tanrikulu AC, Abakay A, Evliyaoglu O, Palanci Y. Coenzyme Q10, copper, zinc, and lipid peroxidation levels in serum of patients with chronic obstructive pulmonary disease. Biol Trace Elem Res. 2011; 143(2):659-667.

3. Dolinay T, Choi AM, Ryter SW. Heme Oxygenase-1/CO as protective mediators in cigarette smoke- induced lung cell injury and chronic obstructive pulmonary disease. Curr Pharm Biotechnol. 2012;13(6): 769-776.

4. Vestbo J, Hurd SS, Agusti AG, et al. Global strategy for the diagnosis, management, and prevention of chronic obstructive pulmonary disease: GOLD executive summary. Am J Respir Crit Care Med. 2013;187(4): 347-365.

5. Trupin L, Earnest G, San Pedro M, et al. The occupational burden of chronic obstructive pulmonary disease. Eur Respir J. 2003;22(3): 462-469.

6. Matheson MC, Benke G, Raven J, et al. Biological dust exposure in the workplace is a risk factor for chronic obstructive pulmonary disease. Thorax. 2005;60(8):645-651.

7. Bernardo I, Bozinovski S, Vlahos R. Targeting oxidant-dependent mechanisms for the treatment of COPD and its comorbidities. Pharmacol Ther. 2015;155:60-79.

8. Ghoorah K, De Soyza A, Kunadian V. Increased cardiovascular risk in patients with chronic obstructive pulmonary disease and the potential mechanisms linking the two conditions: a review. Cardiol Rev. 2013; 21(4):196-202.

9. Bialas AJ, Sitarek P, Milkowska-Dymanowska J, Piotrowski WJ, Gorski P. The role of mitochondria and oxidative/antioxidative imbalance in pathobiology of chronic obstructive pulmonary disease. Oxid Med Cell Longev. 2016;2016:7808576.

10. Puente-Maestu L, Lazaro A, Tejedor A, et al. Effects of exercise on mitochondrial DNA content in skeletal muscle of patients with COPD. Thorax. 2011;66(2):121-127.

11. Yakes FM, Van Houten B. Mitochondrial DNA damage is more extensive and persists longer than nuclear DNA damage in human cells following oxidative stress. Proc Natl Acad Sci US A. 1997;94(2):514-519. 
12. Bernard K, Logsdon NJ, Miguel V, et al. NADPH oxidase 4 (Nox4) suppresses mitochondrial biogenesis and bioenergetics in lung fibroblasts via a nuclear factor erythroid-derived 2-like 2 (Nrf2)-dependent pathway. J Biol Chem. 2017;292(7):3029-3038.

13. Konokhova Y, Spendiff S, Jagoe RT, et al. Failed upregulation of TFAM protein and mitochondrial DNA in oxidatively deficient fibers of chronic obstructive pulmonary disease locomotor muscle. Skelet Muscle. 2016;6:10.

14. Aravamudan B, Thompson MA, Pabelick CM, Prakash YS. Mitochondria in lung diseases. Expert Rev Respir Med. 2013;7(6):631-646.

15. Aravamudan B, Kiel A, Freeman M, et al. Cigarette smoke-induced mitochondrial fragmentation and dysfunction in human airway smooth muscle. Am J Physiol Lung Cell Mol Physiol. 2014;306(9): L840-L854.

16. Hoffmann RF, Zarrintan S, Brandenburg SM, et al. Prolonged cigarette smoke exposure alters mitochondrial structure and function in airway epithelial cells. Respir Res. 2013;14:97.

17. Klaunig JE, Wang $\mathrm{Z}, \mathrm{Pu} \mathrm{X}$, Zhou S. Oxidative stress and oxidative damage in chemical carcinogenesis. Toxicol Appl Pharmacol. 2011; 254(2):86-99.

18. Kanki T, Klionsky DJ. The molecular mechanism of mitochondria autophagy in yeast. Mol Microbiol. 2010;75(4):795-800.

19. Kalyanaraman B. Teaching the basics of redox biology to medical and graduate students: oxidants, antioxidants and disease mechanisms. Redox Biol. 2013;1:244-257.

20. Barbieri E, Sestili P. Reactive oxygen species in skeletal muscle signaling. J Signal Transduct. 2012;2012:e982794.

21. Barbieri E, Sestili P. Reactive oxygen species in skeletal muscle signaling. J Signal Transduct. 2012;2012:982794.

22. Griffith B, Pendyala S, Hecker L, Lee PJ, Natarajan V, Thannickal VJ NOX enzymes and pulmonary disease. Antioxid Redox Signal. 2009; 11(10):2505-2516

23. Pierrou S, Broberg P, O’Donnell RA, et al. Expression of genes involved in oxidative stress responses in airway epithelial cells of smokers with chronic obstructive pulmonary disease. Am J Respir Crit Care Med. 2007; 175(6):577-586.

24. Nagai K, Betsuyaku T, Suzuki M, et al. Dual oxidase 1 and 2 expression in airway epithelium of smokers and patients with mild/moderate chronic obstructive pulmonary disease. Antioxid Redox Signal. 2008, 10(4):705-714.

25. Schneider D, Ganesan S, Comstock AT, et al. Increased cytokine response of rhinovirus-infected airway epithelial cells in chronic obstructive pulmonary disease. Am J Respir Crit Care Med. 2010;182(3):332-340.

26. Pendyala S, Gorshkova IA, Usatyuk PV, et al. Role of Nox 4 and Nox 2 in hyperoxia-induced reactive oxygen species generation and migration of human lung endothelial cells. Antioxid Redox Signal. 2009;11(4): 747-764

27. Zhang X, Shan P, Jiang G, Cohn L, Lee PJ. Toll-like receptor 4 deficiency causes pulmonary emphysema. J Clin Invest. 2006;116(11) 3050-3059.

28. Martyn KD, Frederick LM, von Loehneysen K, Dinauer MC, Knaus UG. Functional analysis of Nox4 reveals unique characteristics compared to other NADPH oxidases. Cell Signal. 2006;18(1):69-82.

29. Pache JC, Carnesecchi S, Deffert C, et al. NOX-4 is expressed in thickened pulmonary arteries in idiopathic pulmonary fibrosis. Nat Med. 2011;17(1):31-32.

30. Xu S, Chamseddine AH, Carrell S, Miller FJ Jr. Nox4 NADPH oxidase contributes to smooth muscle cell phenotypes associated with unstable atherosclerotic plaques. Redox Biol. 2014;2:642-650.

31. Milara J, Peiro T, Serrano A, et al. Roflumilast N-oxide inhibits bronchial epithelial to mesenchymal transition induced by cigarette smoke in smokers with COPD. Pulm Pharmacol Ther. 2014;28(2):138-148.

32. Hollins F, Sutcliffe A, Gomez E, et al. Airway smooth muscle NOX4 is upregulated and modulates ROS generation in COPD. Respir Res. 2016;17(1):84.

33. Liu X, Hao B, Ma A, He J, Liu X, Chen J. The expression of NOX4 in smooth muscles of small airway correlates with the disease severity of COPD. Biomed Res Int. 2016;2016:2891810.
34. Jastroch M, Divakaruni AS, Mookerjee S, Treberg JR, Brand MD. Mitochondrial proton and electron leaks. Essays Biochem. 2010;47: 53-67.

35. Agrawal A, Mabalirajan U. Rejuvenating cellular respiration for optimizing respiratory function: targeting mitochondria. Am J Physiol Lung Cell Mol Physiol. 2016;310(2):L103-L113.

36. Minutoli L, Puzzolo D, Rinaldi M, et al. ROS-Mediated NLRP3 Inflammasome activation in brain, heart, kidney, and testis ischemia/ reperfusion injury. Oxid Med Cell Longev. 2016;2016:2183026.

37. Park J, Choi H, Min JS, et al. Mitochondrial dynamics modulate the expression of pro-inflammatory mediators in microglial cells. J Neurochem. 2013;127(2):221-232.

38. Jiang XL, Zhong P, Huang CL, He F, Fan XM, Chen XR. The relationship between nutritional status and oxidative stress markers, pulmonary function in patients with stable chronic obstructive pulmonary disease. Zhonghua Jie He He Hu Xi Za Zhi. 2017;40(1):40-45. Chinese.

39. van der Toorn M, Rezayat D, Kauffman HF, et al. Lipid-soluble components in cigarette smoke induce mitochondrial production of reactive oxygen species in lung epithelial cells. Am J Physiol Lung Cell Mol Physiol. 2009;297(1):L109-L114.

40. Folz RJ, Guan J, Seldin MF, Oury TD, Enghild JJ, Crapo JD. Mouse extracellular superoxide dismutase: primary structure, tissue-specific gene expression, chromosomal localization, and lung in situ hybridization. Am J Respir Cell Mol Biol. 1997;17(4):393-403.

41. Goldkorn T, Filosto S. Lung injury and cancer: mechanistic insights into ceramide and EGFR signaling under cigarette smoke. Am J Respir Cell Mol Biol. 2010;43(3):259-268.

42. Shapiro SD, Goldstein NM, Houghton AM, Kobayashi DK, Kelley D, Belaaouaj A. Neutrophil elastase contributes to cigarette smoke-induced emphysema in mice. Am J Pathol. 2003;163(6):2329-2335.

43. Moretto N, Bertolini S, Iadicicco C, et al. Cigarette smoke and its component acrolein augment IL-8/CXCL8 mRNA stability via p38 MAPK/MK2 signaling in human pulmonary cells. Am J Physiol Lung Cell Mol Physiol. 2012;303(10):L929-L938.

44. Shapiro SD, Ingenito EP. The pathogenesis of chronic obstructive pulmonary disease: advances in the past 100 years. Am J Respir Cell Mol Biol. 2005;32(5):367-372.

45. Schamberger AC, Staab-Weijnitz CA, Mise-Racek N, Eickelberg O. Cigarette smoke alters primary human bronchial epithelial cell differentiation at the air-liquid interface. Sci Rep. 2015;5:8163.

46. Song YS, Kim MS, Lee DH, Oh DK, Yoon DY. 15-Hydroxyeicosatetraenoic acid inhibits phorbol-12-myristate-13-acetate-induced MUC5AC expression in NCI-H292 respiratory epithelial cells. J Microbiol Biotechnol. 2015;25(5):589-597.

47. Bulua AC, Simon A, Maddipati R, et al. Mitochondrial reactive oxygen species promote production of proinflammatory cytokines and are elevated in TNFR1-associated periodic syndrome (TRAPS). $J$ Exp Med. 2011;208(3):519-533.

48. Ballweg K, Mutze K, Konigshoff M, Eickelberg O, Meiners S. Cigarette smoke extract affects mitochondrial function in alveolar epithelial cells. Am J Physiol Lung Cell Mol Physiol. 2014;307(11):L895-L907.

49. Otera H, Ishihara N, Mihara K. New insights into the function and regulation of mitochondrial fission. Biochim Biophys Acta. 2013;1833(5): 1256-1268.

50. Gomes LC, Di Benedetto G, Scorrano L. During autophagy mitochondria elongate, are spared from degradation and sustain cell viability. Nat Cell Biol. 2011;13(5):589-598.

51. Eura Y, Ishihara N, Oka T, Mihara K. Identification of a novel protein that regulates mitochondrial fusion by modulating mitofusin (Mfn) protein function. J Cell Sci. 2006;119(Pt 23):4913-4925.

52. Zhang J, Liu W, Liu J, et al. G-protein beta2 subunit interacts with mitofusin 1 to regulate mitochondrial fusion. Nat Commun. 2010;1:101.

53. Zorzano A, Liesa M, Palacin M. Mitochondrial dynamics as a bridge between mitochondrial dysfunction and insulin resistance. Arch Physiol Biochem. 2009;115(1):1-12.

54. Ong SB, Kalkhoran SB, Cabrera-Fuentes HA, Hausenloy DJ. Mitochondrial fusion and fission proteins as novel therapeutic targets for treating cardiovascular disease. Eur J Pharmacol. 2015;763(Pt A):104-114. 
55. Chen H, Chomyn A, Chan DC. Disruption of fusion results in mitochondrial heterogeneity and dysfunction. J Biol Chem. 2005;280(28): 26185-26192.

56. Chen H, Detmer SA, Ewald AJ, Griffin EE, Fraser SE, Chan DC. Mitofusins Mfn1 and Mfn2 coordinately regulate mitochondrial fusion and are essential for embryonic development. J Cell Biol. 2003;160(2): 189-200.

57. Song Z, Chen H, Fiket M, Alexander C, Chan DC. OPA1 processing controls mitochondrial fusion and is regulated by mRNA splicing, membrane potential, and Yme1L. J Cell Biol. 2007;178(5): 749-755.

58. Ahmad T, Sundar IK, Lerner CA, et al. Impaired mitophagy leads to cigarette smoke stress-induced cellular senescence: implications for chronic obstructive pulmonary disease. FASEB J. 2015;29(7):2912-2929.

59. Kim KY, Perkins GA, Shim MS, et al. DRP1 inhibition rescues retinal ganglion cells and their axons by preserving mitochondrial integrity in a mouse model of glaucoma. Cell Death Dis. 2015;6:e1839.

60. Wiegman $\mathrm{CH}$, Michaeloudes $\mathrm{C}$, Haji G, et al. Oxidative stress-induced mitochondrial dysfunction drives inflammation and airway smooth muscle remodeling in patients with chronic obstructive pulmonary disease. J Allergy Clin Immunol. 2015;136(3):769-780.

61. Wang K, Yan R, Cooper KF, Strich R. Cyclin C mediates stressinduced mitochondrial fission and apoptosis. Mol Biol Cell. 2015;26(6): 1030-1043

62. Twig G, Shirihai OS. The interplay between mitochondrial dynamics and mitophagy. Antioxid Redox Signal. 2011;14(10):1939-1951.

63. Mizumura K, Cloonan SM, Nakahira K, et al. Mitophagy-dependent necroptosis contributes to the pathogenesis of COPD. $J$ Clin Invest. 2014;124(9):3987-4003.

64. Jin SM, Youle RJ. PINK1 - and Parkin-mediated mitophagy at a glance. J Cell Sci. 2012;125(Pt 4):795-799.

65. Ito S, Araya J, Kurita Y, et al. PARK2-mediated mitophagy is involved in regulation of HBEC senescence in COPD pathogenesis. Autophagy. 2015;11(3):547-559.

66. Chen ZH, Kim HP, Sciurba FC, et al. Egr-1 regulates autophagy in cigarette smoke-induced chronic obstructive pulmonary disease. PLoS One. 2008;3(10):e3316.

67. Kageyama S, Sou YS, Uemura T, et al. Proteasome dysfunction activates autophagy and the Keap1-Nrf2 pathway. J Biol Chem. 2014; 289(36):24944-24955.

68. Shiba-Fukushima K, Imai Y, Yoshida S, et al. PINK1-mediated phosphorylation of the Parkin ubiquitin-like domain primes mitochondrial translocation of Parkin and regulates mitophagy. Sci Rep. 2012; $2: 1002$.
69. Geisler S, Holmstrom KM, Skujat D, et al. PINK1/Parkin-mediated mitophagy is dependent on VDAC1 and p62/SQSTM1. Nat Cell Biol. 2010;12(2):119-131.

70. Gegg ME, Cooper JM, Chau KY, Rojo M, Schapira AH, Taanman JW. Mitofusin 1 and mitofusin 2 are ubiquitinated in a PINK1/parkindependent manner upon induction of mitophagy. Hum Mol Genet. 2010; 19(24):4861-4870

71. Matsumoto G, Wada K, Okuno M, Kurosawa M, Nukina N. Serine 403 phosphorylation of p62/SQSTM1 regulates selective autophagic clearance of ubiquitinated proteins. Mol Cell. 2011;44(2):279-289.

72. Pilli M, Arko-Mensah J, Ponpuak M, et al. TBK-1 promotes autophagymediated antimicrobial defense by controlling autophagosome maturation. Immunity. 2012;37(2):223-234.

73. Ro SH, Semple IA, Park H, et al. Sestrin2 promotes Unc-51-like kinase 1 mediated phosphorylation of p62/sequestosome-1. FEBS J. 2014;281(17):3816-3827.

74. Lim J, Lachenmayer ML, Wu S, et al. Proteotoxic stress induces phosphorylation of p62/SQSTM1 by ULK1 to regulate selective autophagic clearance of protein aggregates. PLoS Genet. 2015;11(2):e1004987.

75. Tran I, Ji C, Ni I, Min T, Tang D, Vij N. Role of cigarette smokeinduced aggresome formation in chronic obstructive pulmonary diseaseemphysema pathogenesis. Am J Respir Cell Mol Biol. 2015;53(2): 159-173.

76. Lippai M, Low P. The role of the selective adaptor p62 and ubiquitinlike proteins in autophagy. Biomed Res Int. 2014;2014:832704

77. Jain A, Lamark T, Sjottem E, et al. p62/SQSTM1 is a target gene for transcription factor NRF2 and creates a positive feedback loop by inducing antioxidant response element-driven gene transcription. J Biol Chem. 2010;285(29):22576-22591.

78. Chang AL, Ulrich A, Suliman HB, Piantadosi CA. Redox regulation of mitophagy in the lung during murine Staphylococcus aureus sepsis. Free Radic Biol Med. 2015;78:179-189.

79. Vij N, Chandramani P, Westphal CV, Hole R, Bodas M. Cigarette smoke induced autophagy-impairment accelerates lung aging, COPDemphysema exacerbations and pathogenesis. Am J Physiol Cell Physiol. Epub 2016 Jul 13.

80. Chen BB, Coon TA, Glasser JR, et al. E3 ligase subunit Fbxo15 and PINK1 kinase regulate cardiolipin synthase 1 stability and mitochondrial function in pneumonia. Cell Rep. 2014;7(2):476-487.

81. Kim SY, Kim HJ, Park MK, et al. Mitochondrial E3 ubiquitin protein ligase 1 mediates cigarette smoke-induced endothelial cell death and dysfunction. Am J Respir Cell Mol Biol. 2016;54(2):284-296.
International Journal of COPD

\section{Publish your work in this journal}

The International Journal of COPD is an international, peer-reviewed journal of therapeutics and pharmacology focusing on concise rapid reporting of clinical studies and reviews in COPD. Special focus is given to the pathophysiological processes underlying the disease, intervention programs, patient focused education, and self management protocols.

\section{Dovepress}

This journal is indexed on PubMed Central, MedLine and CAS. The manuscript management system is completely online and includes a very quick and fair peer-review system, which is all easy to use. Visit http://www.dovepress.com/testimonials.php to read real quotes from published authors. 